\title{
Optimal Decompositions of Barely Separable States
}

\author{
David P. DiVincenzo ${ }^{1}$, Barbara M. Terhal ${ }^{2}$, and Ashish V. Thapliyal ${ }^{3}$ \\ ${ }^{1}$ IBM Research Division, Yorktown Heights, NY 10598, USA, divince@watson.ibm.com \\ ${ }^{2}$ ITF, Universiteit van Amsterdam, Valckenierstraat 65, 1018 XE Amsterdam, and \\ CWI, Kruislaan 413, 1098 SJ Amsterdam, The Netherlands, terhal@wins.uva.nl \\ 3 Dept. of Physics, Univ. of California, Santa Barbara, CA 93106, USA, \\ ash@physics.ucsb.edu
}

(September 3, 2018)

\begin{abstract}
Two families of bipartite mixed quantum states are studied for which it is proved that the number of members in the optimal-decomposition ensemble - the ensemble realizing the entanglement of formation - is greater than the rank of the mixed state. We find examples for which the number of states in this optimal ensemble can be larger than the rank by an arbitrarily large factor. In one case the proof relies on the fact that the partial transpose of the mixed state has zero eigenvalues; in the other case the result arises from the properties of product bases that are completable only by embedding in a larger Hilbert space.
\end{abstract}




\section{INTRODUCTION}

The work of recent years has given us an extensive understanding of the entanglement of pure bipartite quantum states. While there are still many open questions about the entanglement of finite collections of quantum states [1] , a rather complete understanding of 'asymptotic' entanglement, that of a large number of copies of a quantum state, has emerged: a pure state is unentangled if and only if the state can be written in a product form $|\Psi\rangle=\left|\Psi_{A}\right\rangle\left|\Psi_{B}\right\rangle$. The single good quantitative measure of entanglement is $E=S\left(\rho_{A}\right)=$ $S\left(\operatorname{Tr}_{B}|\Psi\rangle\langle\Psi|\right)$, where $S$ is the von Neumann entropy. And, a collection of bipartite pure states with total entanglement $E$ can be reversibly interconverted into any other collection of pure states with entanglement $E$ by purely local operations.

However, despite much recent effort, we cannot claim to have such a complete understanding of quantum entanglement for bipartite mixed states. Its characterization has a remarkably greater complexity and richness than the pure-state case: There is not, except in very simple cases, an unambiguous way to say if a mixed state is entangled or not. There is no single good quantitative measure of mixed-state entanglement. And, it seems that entanglement is irreversibly lost when one attempts to convert it from one mixed-state embodiment to another.

Much of this difficulty can be traced to the basic fact [2. [3] that there is no single way of viewing a mixed quantum state as an ensemble of pure states. In fact, we know that there are infinitely many such representations, and we have previously noted that in general these pure-state ensembles exhibit entirely different entanglement properties. For example, the completely mixed state of two qubits is equally well described as an ensemble of product basis states (no entanglement) or as an ensemble of the four Bell states (all maximally entangled).

It seems that no single measure of entanglement for mixed states is correct, but many different ones are useful depending on the situation. The ensemble decomposition of a mixed state with the maximal entanglement is useful in situations where the two parties 
holding the state can be given aid by a third party to extract a pure state with the greatest entanglement; we have termed the average pure-state entanglement of this ensemble the "entanglement of assistance" [4]. Another, operational characterization of entanglement is the "distillable entanglement" $D$ [5.6], the average number of maximally entangled singlet pairs that can be extracted from many copies of the mixed state by local operations and classical communication. Yet another way of quantifying entanglement related to $D$ has been proposed [7] in which the minimum distance from the set of separable mixed states is taken as the measure of entanglement.

Finally, the entanglement measure on which we focus in this paper is the "entanglement of formation" [5.60. It is the average pure-state entanglement of the ensemble which has minimal entanglement that describes the mixed state. Thus this is dual, in an operational sense, to the entanglement of assistance. It plays several other roles as well: it is converse, in some sense, to the distillable entanglement, in that it gives the number of EPR singlet pairs needed to create the mixed state by local operations. It, like the measure of entanglement in Ref. [7], provides bounds on the distillable entanglement, and thus on other quantities such as the quantum capacity of noisy channels that are of great current interest in quantum information theory.

Thus, we believe that a complete characterization of the mathematical properties of the entanglement of formation should be valuable in the continued development of quantum information theory. In this paper we give new results on one particular feature of the entanglement of formation, the least number of states needed to make up a minimal-entanglement ensemble of a mixed state. (In [8] such optimal decompositions of mixed quantum states have already been considered, but with respect to a function related to, but different from, the entanglement of formation.) Determining such optimal decompositions gives information about the minimal-complexity procedures for creating a mixed state from a supply of EPR singlets. But besides the operational significance of our results, we believe that the characterizations we provide are of greater significance on account of the light they shed on the complexity and richness of the mathematical structure of this important concept in 
quantum information theory.

\section{ENTANGLEMENT BASICS}

Let $\rho$ be a density matrix on the bipartite Hilbert space $\mathcal{H}_{A} \otimes \mathcal{H}_{B}$ and let $\mathcal{E}_{\rho}=\left\{p_{i},\left|\psi_{i}\right\rangle\right\}$ with $p_{i}>0$ be an ensemble into which $\rho$ can be decomposed:

$$
\rho=\sum_{i} p_{i}\left|\psi_{i}\right\rangle\left\langle\psi_{i}\right|
$$

The entanglement of formation [6] of $\rho$ is defined as [9]

$$
E(\rho)=\min _{\mathcal{E}_{\rho}} \sum_{i} p_{i} E\left(\left|\psi_{i}\right\rangle\left\langle\psi_{i}\right|\right)
$$

where

$$
E(|\psi\rangle\langle\psi|)=S\left(\operatorname{Tr}_{A}|\psi\rangle\langle\psi|\right)=S\left(\operatorname{Tr}_{B}|\psi\rangle\langle\psi|\right),
$$

where $S($.$) is the von Neumann entropy:$

$$
S(\rho)=-\operatorname{Tr} \rho \log \rho .
$$

The minimization in Eq. (2) makes an analytical computation of the entanglement of formation of mixed states a nontrivial task. Only in a bipartite Hilbert space $2 \otimes 2$ has the problem of determining the entanglement of formation of any density matrix been completely solved, in the work of Wootters [10]. Uhlmann [8] has shown that every bipartite density matrix $\rho$ admits an optimal decomposition, that is, the one that achieves the entanglement

of formation $E$ of $\rho$ (Eq. (2)), with at least $\mathcal{R}(\rho)$ and at most $\mathcal{R}(\rho)^{2}$ different pure states, where $\mathcal{R}(\rho)$ is the rank of the density matrix. We call the number of different pure states in an ensemble that forms a decomposition of a density matrix $\rho$ the cardinality of the ensemble. We say that the optimal ensemble cardinality of a separable state $\rho$, which we denote by $\mathcal{L}_{\mathcal{E}}(\rho)$, is $k$ if at least $k$ different pure states are required for a separable decomposition of $\rho$. Since the number of states must at least be sufficient to span the support of $\rho$, 


$$
\mathcal{L}_{\mathcal{E}}(\rho) \geq \mathcal{R}(\rho),
$$

directly giving Uhlmann's lower bound. However, it has been a open question whether there are states for which the optimal decomposition has more than $\mathcal{R}(\rho)$ different pure states. Note that the states in the decomposition of a density matrix $\rho$ are always in the range of $\rho$. This means that if $\mathcal{L}_{\mathcal{E}}(\rho)>\mathcal{R}(\rho)$ the states in the optimal decomposition will be linearly dependent.

In this paper we present two sets of examples of separable bipartite states $\rho$ for which we prove that the cardinality of the optimal decomposition of $\rho$ exceeds $\mathcal{R}(\rho)$. These are the first examples of such states. Both types of examples can be found in principle in arbitrary high dimensions.

It is useful to classify states according to their behavior under partial transposition. Let $\rho^{T_{B}}=\left(\mathbf{1}_{A} \otimes T\right) \rho$ where $T$ is transposition of a matrix in a chosen basis. $\rho$ is positive under partial transposition (PPT) if $\rho^{T_{\mathrm{B}}}$ is a density matrix, i.e., it has no negative eigenvalues. If $\rho$ is negative under partial transposition (NPT) then $\rho^{T_{B}}$ has at least one negative eigenvalue. It is known that for $2 \otimes 2$ and $2 \otimes 3$ systems, PPT is a necessary and sufficient condition for separability [11]. For a bipartite state in a Hilbert space of arbitrary dimension, PPT is a necessary condition for separability [12].

\section{SEPARABLE STATES AT THE BOUNDARY}

In this section we show that if a separable state and its partial transpose have unequal ranks, then one of them must have its optimal ensemble cardinality greater than its rank. From this result we prove that partial transposes of full-rank separable states that lie on the boundary of the set of PPT states have optimal ensemble cardinality greater than their ranks. Finally we give examples of such states for any $n \otimes n$ system.

We start with the following straightforward observation:

Lemma 1 Let $\rho$ be a separable state on $\mathcal{H}_{A} \otimes \mathcal{H}_{B}$. Let $\rho^{T_{B}}=\left(\mathbf{1}_{A} \otimes T\right) \rho$ where $T$ is transposition in a chosen basis. Then 


$$
\mathcal{L}_{\mathcal{E}}(\rho)=\mathcal{L}_{\mathcal{E}}\left(\rho^{\mathrm{T}_{\mathrm{B}}}\right) .
$$

Proof We prove that $\mathcal{L}_{\mathcal{E}}\left(\rho^{T_{\mathrm{B}}}\right) \leq \mathcal{L}_{\mathcal{E}}(\rho)$ and $\mathcal{L}_{\mathcal{E}}\left(\rho^{T_{\mathrm{B}}}\right) \geq \mathcal{L}_{\mathcal{E}}(\rho)$. Since $\rho$ is separable, its optimal decomposition involves only product states:

$$
\rho=\sum_{i=1}^{\mathcal{L}_{\mathcal{E}}(\rho)} p_{i}\left|\psi_{i}\right\rangle\left\langle\psi_{i}|\otimes| \phi_{i}\right\rangle\left\langle\phi_{i}\right| .
$$

Then it follows that

$$
\rho^{\mathrm{T}_{\mathrm{B}}}=\sum_{i=1}^{\mathcal{L}_{\mathcal{E}}(\rho)} p_{i}\left|\psi_{i}\right\rangle\left\langle\psi_{i}|\otimes| \phi_{i}^{*}\right\rangle\left\langle\phi_{i}^{*}\right|,
$$

and thus $\mathcal{L}_{\mathcal{E}}\left(\rho^{\mathrm{T}_{\mathrm{B}}}\right)$ is at most $\mathcal{L}_{\mathcal{E}}(\rho)$. By performing the partial transpose again on $\rho^{\mathrm{T}_{\mathrm{B}}}$ we can prove the inequality in the other direction.

We have seen that the optimal ensemble cardinality is invariant under partial transposition. The rank of a density matrix $\rho$ is not necessarily invariant under partial transposition. We can draw the following conclusion:

Theorem 1 Let $\rho$ be a separable state on $\mathcal{H}_{A} \otimes \mathcal{H}_{B}$. If

$$
\mathcal{R}(\rho) \neq \mathcal{R}\left(\rho^{\mathrm{T}_{\mathrm{B}}}\right)
$$

then either $\rho$ has the property that $\mathcal{L}_{\mathcal{E}}(\rho)>\mathcal{R}(\rho)$ or $\rho^{T_{\mathrm{B}}}$ has the property that $\mathcal{L}_{\mathcal{E}}\left(\rho^{\mathrm{T}_{\mathrm{B}}}\right)>$ $\mathcal{R}\left(\rho^{\mathrm{T}_{\mathrm{B}}}\right)$.

Proof This follows directly from Lemma 1 and Eq. (5).

Where do we find separable states $\rho$ with the property Eq. (9)? For this we look at full-rank separable states that lie on the boundary of the set of PPT states. The following lemma, illustrated by Fig. 1, looks into this:

Lemma 2 Let $\rho$ be a separable state on $\mathcal{H}_{A} \otimes \mathcal{H}_{B}$ with full rank, $\mathcal{R}(\rho)=\operatorname{dim} \mathcal{H}_{A} \otimes \mathcal{H}_{B}$. If $\rho$ lies on the boundary of the set of PPT states, then

$$
\mathcal{R}(\rho)>\mathcal{R}\left(\rho^{\mathrm{T}_{\mathrm{B}}}\right) .
$$


Proof The set of PPT states $\mathcal{S}_{\mathrm{PPT}}=\left\{\rho \mid \rho^{\mathrm{T}_{\mathrm{B}}} \geq 0\right\}$ is a closed convex set. For the separable states $\rho$ on the boundary of this set, the state $\rho^{T_{B}}$ has at least one eigenvalue which is zero, as $\rho$ is arbitary close to entangled states $\rho_{E}$ for which $\rho_{E}^{T_{B}}$ has at least one negative eigenvalue. Thus $\rho^{\mathrm{T}_{\mathrm{B}}}$ does not have full rank, and for full-rank states $\rho$ this implies $\mathcal{R}(\rho)>\mathcal{R}\left(\rho^{\mathrm{T}_{\mathrm{B}}}\right)$.

One can remark the following: In $2 \otimes 2$ and $2 \otimes 3$ all entangled density matrices have the property that $\rho^{T_{\mathrm{B}}} \nsucceq 0$ [11]. Therefore any separable density matrix $\rho$ that is on the boundary of the set of separable states and has full rank, fulfills the conditions of Lemma 2. With Theorem 1 it follows that the partial transposes of these density matrices have the desired property, i.e., $\mathcal{L}_{\mathcal{E}}\left(\rho^{\mathrm{T}_{\mathrm{B}}}\right)>\mathcal{R}\left(\rho^{\mathrm{T}_{\mathrm{B}}}\right)$.

A final comment about the results of this section: Eqs. (7) and (8) show that for separable $\rho, \rho=\rho^{\mathrm{T}_{\mathrm{B}}}$ if all the states $\phi_{i}$ are real in some local basis, so obviously the ranks of $\rho$ and $\rho^{T_{\mathrm{B}}}$ are equal. Thus, any state $\rho$ satisfying Lemma 2 must have complex state vectors in any separable decomposition in any local basis. Note that even real density matrices $\rho$ sometimes have optimal decompositions which require complex vectors [13]. Readers may find it surprising that even for complex vectors, there exist sets $\left|\psi_{i}\right\rangle\left|\phi_{i}\right\rangle$ for which the set $\left|\psi_{i}\right\rangle\left|\phi_{i}^{*}\right\rangle$ spans a space of a different dimension; but this is exactly the consequence of Lemma 2 .

\section{A. Examples}

The generalized Werner state [14 in $n \otimes n$ is defined as

$$
\rho_{\mathrm{W}}(f)=f\left|\Psi^{+}\right\rangle\left\langle\Psi^{+}\right|+\frac{1-f}{n^{2}} \mathbf{1}_{n^{2}},
$$

where $\left|\Psi^{+}\right\rangle=\frac{1}{\sqrt{n}} \sum_{i=0}^{n-1}|i i\rangle$ is the maximally entangled state in $n \otimes n$ and $0 \leq f \leq 1$. Let

$$
\rho(f)=\rho_{\mathrm{W}}(f)^{\mathrm{T}_{\mathrm{B}}}, \text { on } \mathcal{H}_{n} \otimes \mathcal{H}_{n} .
$$

It has been shown by Horodecki and Horodecki [14] that the state $\rho_{\mathrm{w}}(f)$ is separable for $0 \leq f \leq 1 / n$. On the other hand, for $1 / n<f \leq 1, \rho(f)$ is not positive semidefinite. 
Therefore the state $\rho_{\mathrm{W}}(1 / n)$ lies at the PPT-NPT boundary. Upon inspection of Eq. (11) we see that the rank of $\rho_{\mathrm{W}}(1 / n)$ is full, $\mathcal{R}\left(\rho_{\mathrm{W}}(1 / n)\right)=n^{2}$. Thus we can use Theorem 1 and Lemma 2 .

It is easy to show by direct calculation that $\rho(1 / n)$ has exactly $n(n-1) / 2$ zero eigenvalues and hence its rank is $n(n+1) / 2$. Therefore by Theorem 1 and Lemma 2, we find that $\rho(1 / n)$ has an optimal ensemble cardinality of at least $n^{2}$ whereas the $\mathcal{R}(\rho(1 / n))=n(n+1) / 2$. For this state, the ratio $\frac{\mathcal{L}_{\mathcal{E}}(\rho(1 / n))}{\mathcal{R}(\rho(1 / n))}$ can be as large as 2 when $n$ tends to $\infty$. This ratio could be made higher if there exists a $\rho$ in $n \otimes n$ for which $\rho^{\mathrm{T}_{\mathrm{B}}}$ can have more than $n(n-1) / 2$ eigenvalues; we have no indication that this is possible. But for the state $\rho(1 / n)^{\otimes k}, k$ tensor copies of the above state, the ratio of $\mathcal{L}_{\mathcal{E}}$ to $\mathcal{R}$ can be made arbitrarily large (going to $2^{k}$ for $n \rightarrow \infty)$.

\section{BARELY COMPLETABLE SETS OF PRODUCT STATES}

In [15,16] the notions of an unextendible product basis and an uncompletable product basis were introduced. A product basis is a set of $k$ separable orthogonal pure states in $n \otimes m$. Considering the case $k<n m$, the basis is unextendible if there are no additional pure product states orthogonal to all the members of the basis; it is uncompletable (in $n \otimes m$ ) if the number of such additional states is less than $n m-k$. In [15,16] it is shown that the completely mixed state $\rho$ on the Hibert space complementary to the space spanned by the unextendible product basis is entangled, but has the property that $\rho^{T_{B}}$ is positive semidefinite. In the following, we will need the notion of a local extension of a bipartite Hilbert space $\mathcal{H}=$ $\mathcal{H}_{A} \otimes \mathcal{H}_{B}$ : a local extension of $\mathcal{H}$ is a Hilbert space $\mathcal{H}^{\prime}=\left(\mathcal{H}_{A} \oplus \mathcal{H}_{A}^{\prime}\right) \otimes\left(\mathcal{H}_{B} \oplus \mathcal{H}_{B}^{\prime}\right)$.

In [15] and [16] the notions of an unextendible product basis and an uncompletable product basis were introduced [17]. It was shown how to construct, from an unextendible product basis, a bipartite entangled state $\rho$ for which $\rho^{T_{\mathrm{B}}}$ is positive semidefinite. We will need the notion of a local extension of a bipartite Hilbert space $\mathcal{H}=\mathcal{H}_{A} \otimes \mathcal{H}_{B}$ : A local extension of $\mathcal{H}$ is a Hilbert space $\mathcal{H}^{\prime}=\left(\mathcal{H}_{A} \oplus \mathcal{H}_{A}^{\prime}\right) \otimes\left(\mathcal{H}_{B} \oplus \mathcal{H}_{B}^{\prime}\right)$. 
Theorem 2 Let $\left\{\left|\alpha_{i}\right\rangle \otimes\left|\beta_{i}\right\rangle\right\}_{i=1}^{|S|}$ be a partial product basis $S$ in $\mathcal{H}=\mathcal{H}_{A} \otimes \mathcal{H}_{B}$. If $S$ is uncompletable in $\mathcal{H}$, but $S$ is completable in some local extension $\mathcal{H}^{\prime}$ of $\mathcal{H}$, then $\rho_{S}$ defined as

$$
\rho_{S}=\frac{1}{\operatorname{dim} \mathcal{H}-|S|}\left(\mathbf{1}-\sum_{i=1}^{|S|}\left|\alpha_{i}\right\rangle\left\langle\alpha_{i}|\otimes| \beta_{i}\right\rangle\left\langle\beta_{i}\right|\right)
$$

has the property that

$$
\mathcal{L}_{\mathcal{E}}\left(\rho_{S}\right)>\mathcal{R}\left(\rho_{S}\right)
$$

Proof As the set of states $S$ is completable in a local extension of $\mathcal{H}$, the state $\rho_{S}$ is separable, by Lemma 2 of [15]. The idea of this Lemma 2 is that the completion of the set $\left\{\left|\alpha_{i}\right\rangle \otimes\left|\beta_{i}\right\rangle\right\}_{i=1}^{|S|}$ in $\mathcal{H}^{\prime}$ give rise to a separable state

$$
\rho_{S}^{\prime}=\frac{1}{\operatorname{dim} \mathcal{H}^{\prime}-|S|}\left(\mathbf{1}^{\prime}-\sum_{i=1}^{|S|}\left|\alpha_{i}\right\rangle\left\langle\alpha_{i}|\otimes| \beta_{i}\right\rangle\left\langle\beta_{i}\right|\right) .
$$

But $\rho_{S}$ is obtained from $\rho_{S}^{\prime}$ by local projections on to $\mathcal{H}_{A}$ and $\mathcal{H}_{B}$ and therefore $\rho_{S}$ is separable as well. However, since $\rho_{S}$ is uncompletable in $\mathcal{H}, \rho_{S}$ cannot be represented as an ensemble of orthogonal product states of cardinality $\mathcal{R}\left(\rho_{S}\right)$. Thus any optimal decomposition of $\rho_{S}$ must use non-orthogonal product states. The von Neumann entropy of $\rho_{S}$ is equal to $S\left(\rho_{S}\right)=\log \mathcal{R}\left(\rho_{S}\right)$ as $\rho_{S}$ is the identity on a space of dimension $\mathcal{R}\left(\rho_{S}\right)$. In order to achieve this entropy, the optimal decomposition of $\rho_{S}$ has to use more than $\mathcal{R}\left(\rho_{S}\right)$ product states, or

$$
\mathcal{L}_{\mathcal{E}}\left(\rho_{S}\right)>\mathcal{R}\left(\rho_{S}\right)
$$

because any density matrix $\rho$ which is a mixture of only $n$ non-orthogonal states has entropy strictly less than $\log n$ bits. 


\section{A. An Example}

In [15] an example was given of a set of orthogonal product states on $3 \otimes 4$ that is not completable in $3 \otimes 4$, but is completable in $3 \otimes 5$. We reproduce the states here: Consider the states $\vec{v}_{i} \otimes \vec{w}_{i}, \quad i=0, \ldots, 4$ with $\vec{v}_{i}$ defined as

$$
\vec{v}_{i}=N\left(\cos \frac{2 \pi i}{5}, \sin \frac{2 \pi i}{5}, h\right), \quad i=0, \ldots, 4
$$

with $h=\frac{1}{2} \sqrt{1+\sqrt{5}}$ and $N=2 / \sqrt{5+\sqrt{5}}$. The states $\vec{w}_{j}$ are defined as

$$
\begin{gathered}
\vec{w}_{j}=N^{\prime}(\sqrt{\cos (\pi / 5)} \cos (2 j \pi / 5), \sqrt{\cos (\pi / 5)} \sin (2 j \pi / 5), \\
\sqrt{\cos (2 \pi / 5)} \cos (4 j \pi / 5), \sqrt{\cos (2 \pi / 5)} \sin (4 j \pi / 5)),
\end{gathered}
$$

with normalization $N^{\prime}=\sqrt{2 / \sqrt{5}}$. Note that $\vec{w}_{j}^{T} \vec{w}_{j+1}=0($ addition $\bmod 5)$. One can show that this set, albeit extendible on $3 \otimes 4$, is not completable: One can at most add three vectors like $\vec{v}_{0} \otimes\left(\vec{w}_{0}, \vec{w}_{1}, \vec{w}_{4}\right)^{\perp}, \vec{v}_{3} \otimes\left(\vec{w}_{2}, \vec{w}_{3}, \vec{w}_{4}\right)^{\perp}$ and $\left(\vec{v}_{0}, \vec{v}_{3}\right)^{\perp} \otimes\left(\vec{w}_{1}, \vec{w}_{2}, \vec{w}_{4}\right)^{\perp}$. The completion of this set in $3 \otimes 5$ is particularly simple, being given by the following ten states:

$$
\begin{aligned}
& \left(\vec{v}_{1}, \vec{v}_{4}\right)^{\perp} \otimes \vec{x}_{0}, \vec{v}_{0} \otimes\left(\vec{w}_{0}^{\perp} \in \operatorname{span}\left(\vec{x}_{4}, \vec{x}_{1}\right)\right), \\
& \left(\vec{v}_{0}, \vec{v}_{2}\right)^{\perp} \otimes \vec{x}_{1}, \vec{v}_{1} \otimes\left(\vec{w}_{1}^{\perp} \in \operatorname{span}\left(\vec{x}_{0}, \vec{x}_{2}\right)\right), \\
& \left(\vec{v}_{1}, \vec{v}_{3}\right)^{\perp} \otimes \vec{x}_{2}, \vec{v}_{2} \otimes\left(\vec{w}_{2}^{\perp} \in \operatorname{span}\left(\vec{x}_{1}, \vec{x}_{3}\right)\right), \\
& \left(\vec{v}_{2}, \vec{v}_{4}\right)^{\perp} \otimes \vec{x}_{3}, \vec{v}_{3} \otimes\left(\vec{w}_{3}^{\perp} \in \operatorname{span}\left(\vec{x}_{2}, \vec{x}_{4}\right)\right), \\
& \left(\vec{v}_{0}, \vec{v}_{3}\right)^{\perp} \otimes \vec{x}_{4}, \vec{v}_{4} \otimes\left(\vec{w}_{4}^{\perp} \in \operatorname{span}\left(\vec{x}_{3}, \vec{x}_{0}\right)\right) .
\end{aligned}
$$

The state $\rho_{S}$ on $3 \otimes 4$ has rank seven, but the separable decomposition consists of ten non-orthogonal states obtained by projecting the orthogonal states of the completion, Eq. (19), back into the $3 \otimes 4$ Hilbert space. It is not known whether there exists a separable decomposition with more than seven but with fewer than ten states.

\section{DISCUSSION}

The results presented here on the minimum cardinality of optimal ensembles raises a large number of tantalizing questions; we would like to know this cardinality for all possible 
mixed states. So far, our rigorous results apply only to separable states. There is some empirical evidence that for entangled mixed states as well (in fact, for states arising in the theory of unextendible product bases), the optimal ensembles can have a cardinality greater than the rank [18]. But we have found no techniques for proving any results for inseparable mixed states. We would also like to know whether there are cases for which the Uhlmann upper bound of $\mathcal{R}(\rho)^{2}$ is attained. The states shown above are still far from this; for the

states at the end of Sec. III A with $n=2$ and any $k, \mathcal{L}_{\mathcal{E}}=\mathcal{R}^{\frac{\log 4}{\log 3}}$. Finally we note that all the rigorous results we have pertain to cases where $\mathcal{L}_{\mathcal{E}}$, while greater than $\mathcal{R}$, never exceeds the Hilbert space dimension. Is there some reason that $\mathcal{L}_{\mathcal{E}}$ can never exceed this dimension? In conclusion, we have shown two different families of unentangled mixed states for which the minimal number of pure states in an optimal minimal-entanglement ensemble is provably greater than the rank of the mixed state. In both cases the proofs are possible because the mixed state is marginally separable, in the first case because the partial transpose of the state has zero eigenvalues, and in the second because the state is defined as the complement of a barely completable product basis.

\section{ACKNOWLEDGMENTS}

We are grateful to Peter Shor for the construction of the barely uncompletable productbasis example used here. We thank Charles Bennett, Richard Jozsa, John Smolin, and Armin Uhlmann for helpful discussions. AVT would like to thank David D. Awschalom for his invaluable support without which it would have been impossible to work in this exciting field. This work has been supported in part by the Army Research Office under contract numbers DAAG55-98-C-0041 and DAAG55-98-1-0366. AVT and BMT would like to thank IBM Research for logistical support during their visits to the IBM Thomas J. Watson Research Center. 


\section{REFERENCES}

[1] See, for example, D. Jonathan and M. B. Plenio, "Entanglement-assisted local manipulation of pure quantum states," quant-ph/9905071.

[2] E. Schrodinger, "Probability relations between separated systems," Proc. Camb. Phil. Soc. 31, 446 (1935).

[3] L. P. Hughston, R. Jozsa, and W. K. Wootters, "A complete classification of quantum ensembles having a given density matrix," Phys. Lett. A 183, 14 (1993).

[4] O. Cohen, Phys. Rev. Lett 80, 2493 (1998); D. P. DiVincenzo, C. A. Fuchs, J. A. Smolin, A. Thapliyal, and A. Uhlmann, "Entanglement of assistance", "Proceedings of the First NASA International Conference on Quantum Computing and Quantum Communications", 17-20th February 1998, Palm Springs, CA, Springer-Verlag Lecture Notes in Computer Science Vol. 1509, (Springer-Verlag, Heidelberg, Germany, 1999) [LANL preprint quant-ph/9803033.

[5] C. H. Bennett, H. J. Bernstein, S. Popescu, B. Schumacher, Phys. Rev. A 53, 2046 (1996) [LANL preprint quant-ph/9511030].

[6] C. H. Bennett, D. P. DiVincenzo, J. A. Smolin, W. K. Wootters, Phys. Rev. A 54, 3824 (1996) [LANL preprint quant-ph/9604024].

[7] V. Vedral, M. B. Plenio, M. A. Rippin, P. L. Knight, Phys. Rev. Lett. 78, 2275 (1997) [LANL preprint quant-ph/9702027]. A more detailed account can be found in V. Vedral and M. Plenio, Phys. Rev. A 57, 1619 (1998) [LANL preprint quant-ph/9707035.

[8] A. Uhlmann, Open Sys. \& Information Dyn. 5, 209 (1998).

[9] It has been pointed out that a more satisfactory definition of the entanglement of formation would be

$$
E(\rho)=\min _{N, \mathcal{E}_{\rho} \otimes N} \frac{1}{N} \sum_{i} p_{i} E\left(\left|\psi_{i}\right\rangle\left\langle\psi_{i}\right|\right)
$$


Eqs. (20) and (2) are equal if the entanglement of formation is additive, but this property has not been proved. The analysis of this paper concerns the original definition of entanglement of formation, Eq. (2).

[10] W. K. Wootters, Phys. Rev. Lett. 80, 2245 (1998) [LANL preprint quant-ph/9709029].

[11] R. Horodecki, M. Horodecki, and P. Horodecki, Phys. Lett. A 223, 1 (1996).

[12] A. Peres, Phys. Rev. Lett. 76, 1413 (1996), [LANL preprint quant-ph/9604005].

[13] Eq.(30) of [6] gives a decomposition with eight members for any mixture of Bell states. However it can be easily seen that appropriate sets of four of these give optimalcardinality ensembles with at least one complex vector.

[14] M. Horodecki and P. Horodecki, "Reduction criterion of separability and limits for a class of protocols of entanglement distillation," [LANL preprint quant-ph/9708015].

[15] C. H. Bennett, D. P. DiVincenzo, T. Mor, P. W. Shor, J. A. Smolin and B. M. Terhal, "Unextendible Product Bases and Bound Entanglement," [LANL preprint quant-ph/9808030.

[16] C. H. Bennett, D. P. DiVincenzo, T. Mor, P. W. Shor, J. A. Smolin and B. M. Terhal, "Unextendible Product Bases and Bound Entanglement II," in preparation.

[17] A product basis is a set of $k$ separable orthogonal pure states in $n \otimes m$. Considering the case $k<n m$, the basis is unextendible if these are no additional pure product states orthogonal to all the members of the basis; it is uncompletable (in $n \otimes m$ ) if the number of such additional states is less than $n m-k$.

[18] J. A. Smolin (private communication). 


\section{FIGURES}

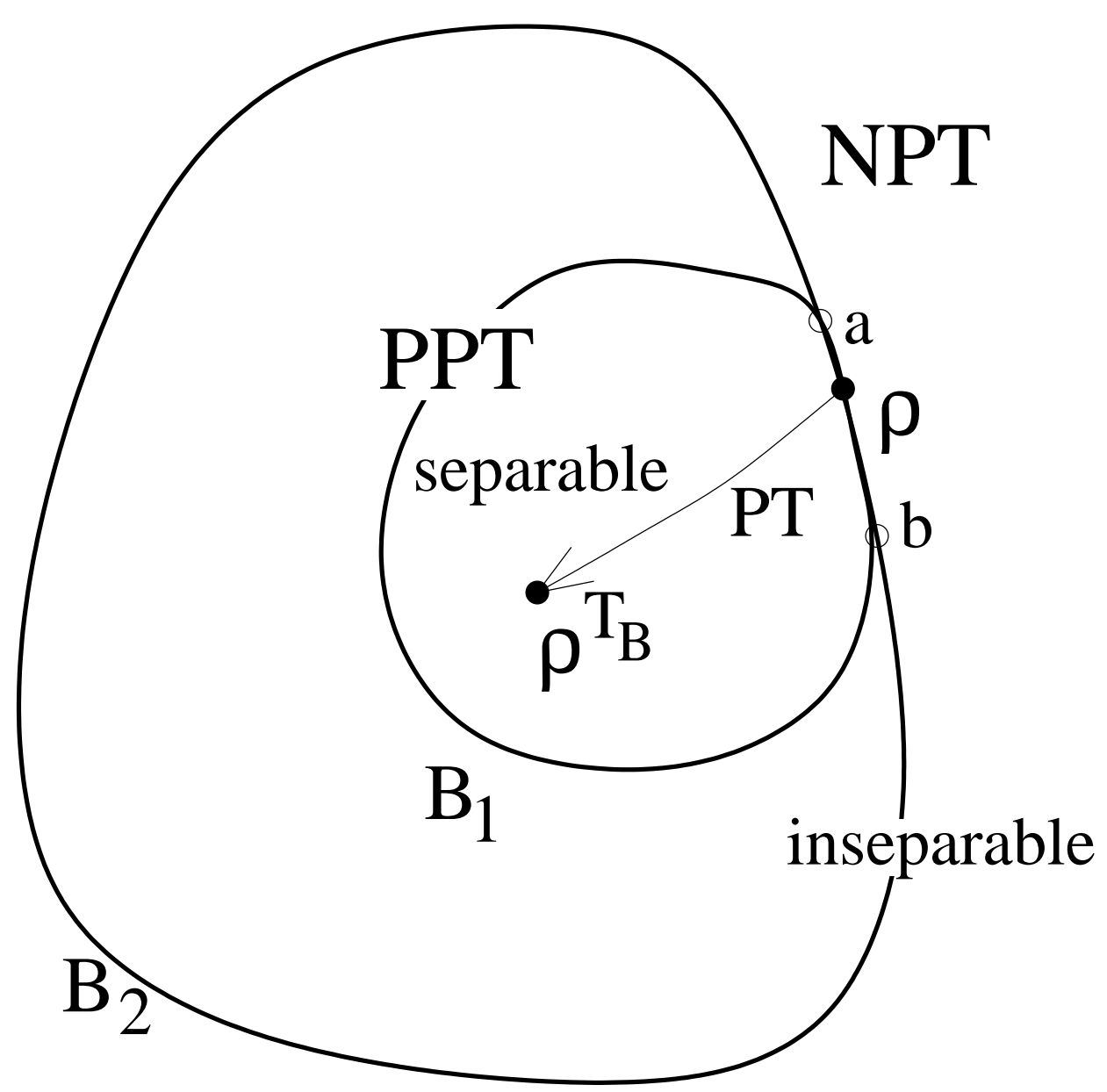

FIG. 1. Illustration of the construction of Lemma 2. The PPT, NPT, separable, and inseparable sets of density matrices are indicated. $B_{1}$ is the boundary between separable and inseparable, $B_{2}$ between PPT and NPT. For $2 \otimes 2$ and $2 \otimes 3, B_{1}=B_{2}$, but in general they do not coincide. The conditions of the Lemma are met when $\rho$ has full rank, is separable, and is on the boundary $B_{2}$ so that its partial transpose $\rho^{T_{\mathrm{B}}}$ has some zero eigenvalues. $\rho$ must therefore be at a place where $B_{1}$ and $B_{2}$ coincide, such as the arc $a b . \rho^{\mathrm{T}_{\mathrm{B}}}$ is separable; also, it cannot be on the boundary $B_{2}$, because its partial transpose, which is $\rho$, has strictly positive eigenvalues. However, it is possible that $\rho^{T_{\mathrm{B}}}$ could sit on the boundary $B_{1}$ where $B_{1}$ and $B_{2}$ do not coincide. 